\title{
Hamiltonian Formulation of 2 Bounded Immiscible Media with Constant Non-Zero Vorticities and a Common Interface
}

\author{
Alan Compelli \\ Technological University Dublin, alan.compelli@tudublin.ie
}

Follow this and additional works at: https://arrow.tudublin.ie/scschmatart

Part of the Mathematics Commons, Non-linear Dynamics Commons, and the Partial Differential Equations Commons

\section{Recommended Citation}

Compelli, A. (2014) Hamiltonian Formulation of 2 Bounded Immiscible Media with Constant Non-Zero Vorticities and a Common Interface, Wave Motion, to appear. doi: 10.21427/8q5f-2e54

This Article is brought to you for free and open access by the School of Mathematics at ARROW@TU Dublin. It has been accepted for inclusion in Articles by an authorized administrator of ARROW@TU Dublin. For more information, please contact arrow.admin@tudublin.ie, aisling.coyne@tudublin.ie,gerard.connolly@tudublin.ie.

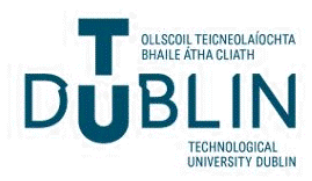




\title{
Hamiltonian Formulation of 2 Bounded Immiscible Media with Constant Non-Zero Vorticities and a Common Interface
}

\author{
Alan Compelli
}

November 30, 2014

\begin{abstract}
We examine a 2-dimensional water-wave system, with gravitationally induced waves, consisting of a lower medium bound underneath by an impermeable flat bed and an upper medium bound above by an impermeable lid such that the 2 media have a free common interface. Both media have constant density and constant (non-zero) vorticity. By examining the governing equations of the system we calculate the Hamiltonian of the system in terms of it's conjugate variables and perform a variable transformation to show that it has canonical Hamiltonian structure.
\end{abstract}

\section{Introduction}

In 1968 Zakharov published a paper [1] showing the canonical Hamiltonian structure of an infinitely deep irrotational fluid system, i.e. with zero vorticity, with a free surface with gravitationally induced waves. Further relevant studies of the irrotational case were carried out in [2], [3], [4], [5], [6]. At the beginning of the 19th century Gernstner [7] had studied vorticity and more recently there have been several papers of interest which consider the rotational case, i.e. with non-zero constant vorticity, e.g. [8], [9], [10], [11], [12], [13], [14], [15], [16], [17]. In particular Constantin et al. [18] showed that a consideration of non-zero vorticity gives a nearly Hamiltonian structure 


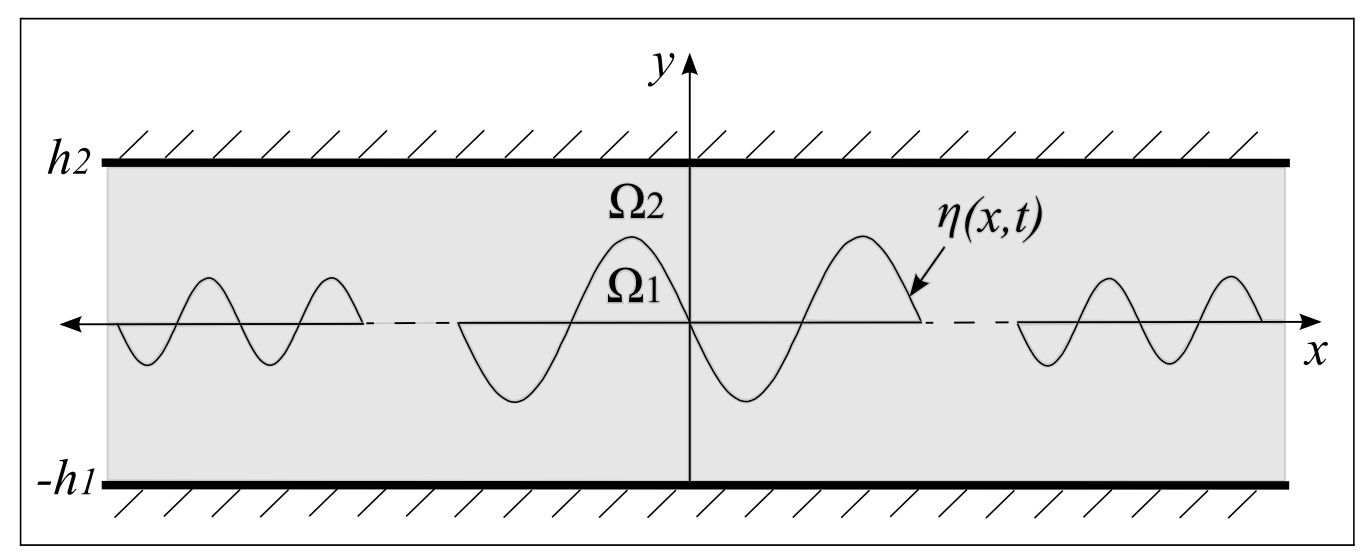

Figure 1: The system under study

(with a linear dependency on a vorticity term). Wahlén [19] then showed that, in fact, the system does indeed have fully Hamiltonian structure, which can be transformed into canonical form.

A consideration of a system consisting of 2 unbounded media with a free common interface was given by Benjamin and Bridges [20], [21]. Craig et al. [22], [23] considered an irrotational system consisting of a lower medium bound underneath by a flat bed and an upper medium bound above by an impermeable lid such that the 2 media have a free common interface and also the case in which the upper media itself has a free surface. The aim of this paper is to show that, in the rotational case, the 2 media bounded system has canonical Hamiltonian structure.

\section{Preliminaries}

As per Figure 1 we define the lower medium $\Omega_{1}$ as the domain $\left\{(x, y) \in \mathbb{R}^{2}\right.$ : $\left.-h_{1}<y<\eta(x, t)\right\}$, the upper medium $\Omega_{2}$ as the domain $\left\{(x, y) \in \mathbb{R}^{2}\right.$ : $\left.\eta(x, t)<y<h_{2}\right\}$ and the entire system $\Omega$ as the domain $\left\{(x, y) \in \mathbb{R}^{2}\right.$ : $\left.-h_{1}<y<h_{2}\right\}$ where $\{y=\eta(x, t)\}$ describes the elevation of the common interface. The subscript $c$ will be used to denote evaluation at the common interface.

We use the subscript notation $i=\{1,2\}$ to represent the lower and upper media respectively and thus can consider a velocity potential $\varphi_{i}$ which is 
defined by:

$$
\left\{\begin{array}{l}
u_{i}=\partial_{x} \varphi_{i}-\omega_{i} y \\
v_{i}=\partial_{y} \varphi_{i}
\end{array}\right.
$$

where non-lateral velocity flow, with propagation in the positive $x$-direction, is given by $\mathbf{V}_{\mathbf{i}}(x, y, z)=\left(u_{i}, v_{i}, 0\right)$ and $\omega_{1}$ and $\omega_{2}$ are the respective non-zero constant vorticities.

Additionally, the stream function $\psi_{i}$ is introduced, defined by:

$$
\left\{\begin{array}{l}
u_{i}=-\partial_{y} \psi_{i} \\
v_{i}=\partial_{x} \psi_{i}
\end{array}\right.
$$

$\rho_{1}$ and $\rho_{2}$ are the respective constant densities of the lower and upper media and stability is given by the condition that $\rho_{1}>\rho_{2}$.

We assume that for large $|x|$ the amplitude of $\eta$ attenuates and hence make the following assumptions

$$
\begin{gathered}
\lim _{|x| \rightarrow \infty} \eta(x, t)=0, \\
\lim _{|x| \rightarrow \infty} \varphi_{i}(x, y, t)=0,
\end{gathered}
$$

and

$$
-h_{1}<\eta(x, t)<h_{2} \text { for all } x \text { and } t \text {. }
$$

\section{Governing Equations}

We write Euler's momentum-conserving equation as:

$$
\partial_{t} \mathbf{V}_{\mathbf{i}}+\left(\mathbf{V}_{\mathbf{i}} \cdot \nabla\right) \mathbf{V}_{\mathbf{i}}=-\frac{1}{\rho_{i}} \nabla P_{i}+\mathbf{g}
$$

where $P_{i}=\rho_{i} g y+p_{\text {atm }}+p_{i}$ is the pressure at a depth $y, p_{\text {atm }}$ is (constant) atmospheric pressure, $p_{i}$ is the dynamic pressure due to the wave motion, $g$ is the acceleration due to gravity (where $y$ points in the opposite direction to the center of gravity) and $\mathbf{g}$ is the force due to gravity per unit mass.

Applying Equations (1) and (2) this can be written as

$$
\nabla\left(\partial_{t} \varphi_{i}+\frac{1}{2}\left(\nabla \psi_{i}\right)^{2}-\omega_{i} \psi_{i}\right)=\nabla\left(-g y-\frac{p_{i}}{\rho_{i}}\right)
$$


where $\nabla=\left(\partial_{x}, \partial_{y}\right)$.

At the interface $p_{1}=p_{2}=p_{c}$ therefore we write Euler's equation in terms of the velocity potentials, stream functions, densities and vorticities as the energy conserving equality

$$
\begin{aligned}
\rho_{1} \nabla\left(\left(\partial_{t} \varphi_{1}\right)_{c}+\frac{1}{2}\left(\nabla \psi_{1}\right)_{c}^{2}\right. & \left.-\omega_{1} \chi_{1}+g \eta\right) \\
& -\rho_{2} \nabla\left(\left(\partial_{t} \varphi_{2}\right)_{c}+\frac{1}{2}\left(\nabla \psi_{2}\right)_{c}^{2}-\omega_{2} \chi_{2}+g \eta\right)=0,
\end{aligned}
$$

where $\chi_{i}$ is the stream function evaluated at the interface.

The following Bernoulli condition at the interface follows from assumptions (3) and (4):

$\rho_{1}\left(\left(\partial_{t} \varphi_{1}\right)_{c}+\frac{1}{2}\left(\nabla \psi_{1}\right)_{c}^{2}-\omega_{1} \chi_{1}+g \eta\right)=\rho_{2}\left(\left(\partial_{t} \varphi_{2}\right)_{c}+\frac{1}{2}\left(\nabla \psi_{2}\right)_{c}^{2}-\omega_{2} \chi_{2}+g \eta\right)$.

We will also use the following kinematic boundary conditions

$$
\left\{\begin{array}{l}
\partial_{t} \eta-\partial_{x} \eta\left(\omega_{i} \eta-\left(\partial_{x} \varphi_{i}\right)_{c}\right)-\left(\partial_{y} \varphi_{i}\right)_{c}=0 \\
\partial_{y}\left(\varphi_{1}\right)_{b}=\partial_{y}\left(\varphi_{2}\right)_{l}=0
\end{array}\right.
$$

noting that $\mathbf{V}_{\mathbf{1}}\left(x,-h_{1}, 0\right)=\left(u_{1}, 0,0\right)$ and $\mathbf{V}_{\mathbf{2}}\left(x, h_{2}, 0\right)=\left(u_{2}, 0,0\right)$, where the subscripts $b$ and $l$ denote evaluation at the bottom (lower boundary) and lid (upper boundary) respectively.

\section{Hamiltonian of the System}

If we consider the system under study as an irrotational system the Hamiltonian, $H$, is given by the sum of the kinetic and potential energies as:

$$
H=\frac{1}{2} \int_{\mathbb{R}} \int_{-h_{1}}^{\eta} \rho_{1}\left(\nabla \varphi_{1}\right)^{2} d y d x+\frac{1}{2} \int_{\mathbb{R}} \int_{\eta}^{h_{2}} \rho_{2}\left(\nabla \varphi_{2}\right)^{2} d y d x+\frac{1}{2} \int_{\mathbb{R}}\left(\rho_{1}-\rho_{2}\right) g \eta^{2} d x .
$$


However, as we are concerned with the rotational case the Hamiltonian (noting the additional vorticity related terms) is given by:

$$
\begin{gathered}
H=\frac{1}{2} \int_{\mathbb{R}} \int_{-h_{1}}^{\eta} \rho_{1}\left(\nabla \varphi_{1}\right)^{2} d y d x+\frac{1}{2} \int_{\mathbb{R}} \int_{\eta}^{h_{2}} \rho_{2}\left(\nabla \varphi_{2}\right)^{2} d y d x+\frac{1}{2} \int_{\mathbb{R}}\left(\rho_{1}-\rho_{2}\right) g \eta^{2} d x \\
-\int_{\mathbb{R}} \int_{-h_{1}}^{\eta} \rho_{1} \omega_{1} y \partial_{x} \varphi_{1} d y d x-\iint_{\mathbb{R}}^{h_{2}} \rho_{2} \omega_{2} y \partial_{x} \varphi_{2} d y d x \\
+\frac{1}{2} \int_{\mathbb{R}} \int_{-h_{1}}^{\eta} \rho_{1} \omega_{1}^{2} y^{2} d y d x+\frac{1}{2} \int_{\mathbb{R}} \int_{\eta}^{h_{2}} \rho_{2} \omega_{2}^{2} y^{2} d y d x .
\end{gathered}
$$

Using the Gauss-Green theorem, and introducing $\xi_{i}:=\left(\varphi_{i}\right)_{c}=\varphi_{i}(x, \eta(x, t), t)$ as the interface velocity potential, we can write the first 2 terms of (12) as

$$
\begin{aligned}
& \frac{1}{2} \int_{\mathbb{R}} \int_{-h_{1}}^{\eta} \rho_{1}\left(\nabla \varphi_{1}\right)^{2} d y d x+\frac{1}{2} \int_{\mathbb{R}} \int_{\eta}^{h_{2}} \rho_{2}\left(\nabla \varphi_{2}\right)^{2} d y d x \\
& \quad=\frac{1}{2} \int_{\mathbb{R}} \rho_{1} \xi_{1} \partial_{\mathbf{n}_{1}} \varphi_{1} \sqrt{1+\left(\partial_{x} \eta\right)^{2}} d x-\frac{1}{2} \int_{\mathbb{R}} \rho_{2} \xi_{2} \partial_{\mathbf{n}_{2}} \varphi_{2} \sqrt{1+\left(\partial_{x} \eta\right)^{2}} d x
\end{aligned}
$$

where $\partial_{\mathbf{n}_{i}} \varphi_{i}$ is the normal derivative of the velocity potential $\varphi_{i}$, at the surface, for an outward normal $\mathbf{n}_{i}$.

Next, we introduce the Dirichlet-Neumann operator $G_{i}(\eta)$ given by (see [3], [23])

$$
G_{i}(\eta) \xi_{i}=\partial_{\mathbf{n}_{i}} \varphi_{i} \sqrt{1+\left(\partial_{x} \eta\right)^{2}}
$$

and hence we can write the first 2 (kinetic energy) terms of (12) as

$$
\begin{aligned}
\frac{1}{2} \int_{\mathbb{R}} \int_{-h_{1}}^{\eta} \rho_{1}\left(\nabla \varphi_{1}\right)^{2} d y d x & +\frac{1}{2} \int_{\mathbb{R}} \int_{\eta}^{h_{2}} \rho_{2}\left(\nabla \varphi_{2}\right)^{2} d y d x \\
& =\frac{1}{2} \int_{\mathbb{R}} \rho_{1} \xi_{1} G_{1}(\eta) \xi_{1} d x+\frac{1}{2} \int_{\mathbb{R}} \rho_{2} \xi_{2} G_{2}(\eta) \xi_{2} d x .
\end{aligned}
$$


Using the kinematic boundary conditions from (10)

$$
\left\{\begin{array}{l}
G_{1}(\eta) \xi_{1}=-\partial_{x} \eta\left(\partial_{x} \varphi_{1}\right)_{c}+\left(\partial_{y} \varphi_{1}\right)_{c}=\partial_{t} \eta-\omega_{1} \eta \partial_{x} \eta \\
G_{2}(\eta) \xi_{2}=\partial_{x} \eta\left(\partial_{x} \varphi_{2}\right)_{c}+\left(\partial_{y} \varphi_{2}\right)_{c}=-\partial_{t} \eta+\omega_{2} \eta \partial_{x} \eta
\end{array}\right.
$$

we get

$$
G_{1}(\eta) \xi_{1}+G_{2}(\eta) \xi_{2}=\left(\omega_{2}-\omega_{1}\right) \eta \partial_{x} \eta
$$

We introduce [20], [21]

$$
\xi:=\rho_{1} \xi_{1}-\rho_{2} \xi_{2}
$$

and hence we can write

$$
\left(\rho_{1} G_{2}(\eta)+\rho_{2} G_{1}(\eta)\right) \xi_{2}=-G_{1}(\eta) \xi+\rho_{1}\left(\omega_{2}-\omega_{1}\right) \eta \partial_{x} \eta .
$$

Also, we introduce

$$
B:=\rho_{1} G_{2}(\eta)+\rho_{2} G_{1}(\eta)
$$

and thus we can write

$$
\left\{\begin{array}{l}
\xi_{1}=B^{-1}\left(G_{2}(\eta) \xi+\rho_{2}\left(\omega_{2}-\omega_{1}\right) \eta \partial_{x} \eta\right) \\
\xi_{2}=B^{-1}\left(-G_{1}(\eta) \xi+\rho_{1}\left(\omega_{2}-\omega_{1}\right) \eta \partial_{x} \eta\right) .
\end{array}\right.
$$

Using (17) the first 2 terms of (12) become

$$
\begin{aligned}
\frac{1}{2} \int_{\mathbb{R}} \rho_{1} \xi_{1} G_{1}(\eta) \xi_{1} d x+\frac{1}{2} \int_{\mathbb{R}} \rho_{2} \xi_{2} G_{2}(\eta) \xi_{2} d x \\
=\frac{1}{2} \int_{\mathbb{R}} \rho_{1} \xi_{1} G_{1}(\eta) \xi_{1} d x+\frac{1}{2} \int_{\mathbb{R}} \rho_{2} \xi_{2}\left(\left(\omega_{2}-\omega_{1}\right) \eta \partial_{x} \eta-G_{1}(\eta) \xi_{1}\right) d x \\
=\frac{1}{2} \int_{\mathbb{R}} \xi G_{1}(\eta) \xi_{1} d x+\frac{1}{2} \int_{\mathbb{R}} \rho_{2}\left(\omega_{2}-\omega_{1}\right) \xi_{2} \eta \partial_{x} \eta d x,
\end{aligned}
$$

and inserting the expressions for $\xi_{1}$ and $\xi_{2}$ from (21) we obtain

$$
\begin{gathered}
=\frac{1}{2} \int_{\mathbb{R}} \xi\left(G_{1}(\eta) B^{-1} G_{2}(\eta)\right) \xi d x+\frac{1}{2} \int_{\mathbb{R}} \rho_{2}\left(\omega_{2}-\omega_{1}\right) \xi G_{1}(\eta) B^{-1} \eta \partial_{x} \eta d x \\
-\frac{1}{2} \int_{\mathbb{R}} \rho_{2}\left(\omega_{2}-\omega_{1}\right) B^{-1} G_{1}(\eta) \xi \eta \partial_{x} \eta d x+\frac{1}{2} \int_{\mathbb{R}} \rho_{1} \rho_{2}\left(\omega_{2}-\omega_{1}\right)^{2} \eta \partial_{x} \eta B^{-1} \eta \partial_{x} \eta d x .
\end{gathered}
$$


We can cancel the second and third terms because the operators $G_{1}$ and $B$ are self-adjoint [22], [23], therefore

$$
\begin{aligned}
& \frac{1}{2} \int_{\mathbb{R}} \int_{-h_{1}}^{\eta} \rho_{1}\left(\nabla \varphi_{1}\right)^{2} d y d x+\frac{1}{2} \int_{\mathbb{R}} \int_{\eta}^{h_{2}} \rho_{2}\left(\nabla \varphi_{2}\right)^{2} d y d x \\
& =\frac{1}{2} \int_{\mathbb{R}} \xi\left(G_{1}(\eta) B^{-1} G_{2}(\eta)\right) \xi d x+\frac{1}{2} \int_{\mathbb{R}} \rho_{1} \rho_{2}\left(\omega_{2}-\omega_{1}\right)^{2} \eta \partial_{x} \eta B^{-1} \eta \partial_{x} \eta d x .
\end{aligned}
$$

Next, we will re-write terms 4 and 5 of (12) using the following lemma.

Lemma 1. For a function $F(x, y)$ which is continuous over $\Omega$ with $h(x)$ defined as follows

$$
h(x)=\int_{0}^{\eta(x)} F(x, y) d y
$$

the derivative with respect to $x$ can be expressed as [18]

$$
h^{\prime}(x)=\int_{0}^{\eta(x)} F_{x}(x, y) d y+F[x, \eta] \partial_{x} \eta .
$$

Using this lemma we let $F=y \varphi_{1}$ and hence

$$
\int_{-h_{1}}^{\eta} y \partial_{x} \varphi_{1} d y=\partial_{x}\left[\int_{-h_{1}}^{\eta} y \varphi_{1} d y\right]-\xi_{1} \eta \partial_{x} \eta
$$

The first term on the right-hand side is zero due to assumption (4). Similarly (noting the sign difference on the right-hand side as $\eta$ is the lower limit for $\left.\Omega_{2}\right)$

$$
\int_{\eta}^{h_{2}} y \partial_{x} \varphi_{2} d y=\xi_{2} \eta \partial_{x} \eta
$$


Therefore

$$
\begin{aligned}
-\int_{\mathbb{R}} \int_{-h_{1}}^{\eta} \rho_{1} \omega_{1} y \partial_{x} \varphi_{1} d y d x-\int_{\mathbb{R}} \int_{\eta}^{h_{2}} \rho_{2} \omega_{2} y \partial_{x} \varphi_{2} d y d x \\
=\int_{\mathbb{R}}\left(\rho_{1} \omega_{1} \xi_{1} \eta \partial_{x} \eta-\rho_{2} \omega_{2} \xi_{2} \eta \partial_{x} \eta\right) d x .
\end{aligned}
$$

Now, inserting the expressions for $\xi_{1}$ and $\xi_{2}$ from (21) gives

$$
\begin{gathered}
\rho_{1} \omega_{1} \xi_{1} \eta \partial_{x} \eta-\rho_{2} \omega_{2} \xi_{2} \eta \partial_{x} \eta=\rho_{1} \omega_{1} B^{-1}\left(G_{2}(\eta) \xi+\rho_{2}\left(\omega_{2}-\omega_{1}\right) \eta \partial_{x} \eta\right) \eta \partial_{x} \eta \\
-\rho_{2} \omega_{2} B^{-1}\left(-G_{1}(\eta) \xi+\rho_{1}\left(\omega_{2}-\omega_{1}\right) \eta \partial_{x} \eta\right) \eta \partial_{x} \eta .
\end{gathered}
$$

Expanding this out gives

$$
\begin{aligned}
\rho_{1} \omega_{1} \xi_{1} \eta \partial_{x} \eta-\rho_{2} \omega_{2} \xi_{2} \eta \partial_{x} \eta=\eta \partial_{x} \eta B^{-1} & \left(\rho_{1} \omega_{1} G_{2}(\eta) \xi+\rho_{2} \omega_{2} G_{1}(\eta) \xi\right) \\
& -\rho_{1} \rho_{2}\left(\omega_{2}-\omega_{1}\right)^{2} \eta \partial_{x} \eta B^{-1} \eta \partial_{x} \eta
\end{aligned}
$$

Therefore

$$
\begin{aligned}
& -\int_{\mathbb{R}} \int_{-h_{1}}^{\eta} \rho_{1} \omega_{1} y \partial_{x} \varphi_{1} d y d x-\int_{\mathbb{R}} \int_{\eta}^{h_{2}} \rho_{2} \omega_{2} y \partial_{x} \varphi_{2} d y d x \\
= & \int_{\mathbb{R}}\left(\eta \partial_{x} \eta B^{-1}\left(\rho_{1} \omega_{1} G_{2}(\eta) \xi+\rho_{2} \omega_{2} G_{1}(\eta) \xi\right)-\rho_{1} \rho_{2}\left(\omega_{2}-\omega_{1}\right)^{2} \eta \partial_{x} \eta B^{-1} \eta \partial_{x} \eta\right) d x .
\end{aligned}
$$

Finally, we write the final 2 terms of (12) as

$$
\frac{1}{2} \int_{\mathbb{R}} \int_{-h_{1}}^{\eta} \rho_{1} \omega_{1}^{2} y^{2} d y d x+\frac{1}{2} \int_{\mathbb{R}} \int_{\eta}^{h_{2}} \rho_{2} \omega_{2}^{2} y^{2} d y d x=\frac{1}{6} \int_{\mathbb{R}}\left(\rho_{1} \omega_{1}^{2}-\rho_{2} \omega_{2}^{2}\right) \eta^{3} d x
$$

Therefore, by substituting (23), (29) and (30) into the expression for the Hamiltonian given in (12) we get the Hamiltonian of the system in terms of the conjugate variables $(\eta, \xi)$ (noting the combination of the second terms in 
(23) and (29))

$$
\begin{gathered}
H(\eta, \xi)=\frac{1}{2} \int_{\mathbb{R}} \xi\left(G_{1}(\eta) B^{-1} G_{2}(\eta)\right) \xi d x+\frac{1}{2} \int_{\mathbb{R}}\left(\rho_{1}-\rho_{2}\right) g \eta^{2} d x \\
-\frac{1}{2} \int_{\mathbb{R}} \rho_{1} \rho_{2}\left(\omega_{2}-\omega_{1}\right)^{2} \eta \partial_{x} \eta B^{-1} \eta \partial_{x} \eta d x \\
+\int_{\mathbb{R}} \eta \partial_{x} \eta B^{-1}\left(\rho_{1} \omega_{1} G_{2}(\eta) \xi+\rho_{2} \omega_{2} G_{1}(\eta) \xi\right) d x+\frac{1}{6} \int_{\mathbb{R}}\left(\rho_{1} \omega_{1}^{2}-\rho_{2} \omega_{2}^{2}\right) \eta^{3} d x .
\end{gathered}
$$

\section{$5 \quad$ Hamiltonian Equations of Motion}

Using the Hamiltonian in (12) the varied Hamiltonian is given by

$$
\begin{gathered}
\delta H=\int_{\mathbb{R}} \int_{-h_{1}}^{\eta} \rho_{1}\left(\nabla \varphi_{1}\right) \cdot \nabla \delta \varphi_{1} d y d x+\int_{\mathbb{R}} \int_{\eta}^{h_{2}} \rho_{2}\left(\nabla \varphi_{2}\right) \cdot \nabla \delta \varphi_{2} d y d x \\
+\frac{1}{2} \int_{\mathbb{R}} \rho_{1}\left(\nabla \varphi_{1}\right)_{c}^{2} \delta \eta d x-\frac{1}{2} \int_{\mathbb{R}} \rho_{2}\left(\nabla \varphi_{2}\right)_{c}^{2} \delta \eta d x+\int_{\mathbb{R}}\left(\rho_{1}-\rho_{2}\right) g \eta \delta \eta d x \\
-\int_{\mathbb{R}} \int_{-h_{1}}^{\eta} \rho_{1} \omega_{1} y \delta\left(\partial_{x} \varphi_{1}\right) d y d x-\int_{\mathbb{R}} \int_{\eta}^{h_{2}} \rho_{2} \omega_{2} y \delta\left(\partial_{x} \varphi_{2}\right) d y d x \\
-\int_{\mathbb{R}}\left(\rho_{1} \omega_{1} \eta\left(\partial_{x} \varphi_{1}\right)_{c}-\rho_{2} \omega_{2} \eta\left(\partial_{x} \varphi_{2}\right)_{c}\right) \delta \eta d x+\frac{1}{2} \int_{\mathbb{R}}\left(\rho_{1} \omega_{1}^{2} \eta^{2}-\rho_{2} \omega_{2}^{2} \eta^{2}\right) \delta \eta d x .
\end{gathered}
$$


Applying Lemma 1 to the following $\left(\Omega_{1}\right)$ term in the varied Hamiltonian gives

$$
\begin{aligned}
& -\int_{\mathbb{R}} \int_{-h_{1}}^{\eta} \rho_{1} \omega_{1} y \delta\left(\partial_{x} \varphi_{1}\right) d y d x \\
& \quad=-\int_{\mathbb{R}} \partial_{x}\left[\int_{-h_{1}}^{\eta} \rho_{1} \omega_{1} y \delta \varphi_{1} d y\right] d x+\int_{\mathbb{R}} \rho_{1} \omega_{1} \eta \partial_{x} \eta\left(\delta \varphi_{1}\right)_{c} d x .
\end{aligned}
$$

The first term on the right-hand side is zero as $\left.\delta \varphi_{i}\right|_{|x| \rightarrow \infty}=0$ since $\left(\varphi_{i}\right)_{c} \rightarrow 0$ as $|x| \rightarrow \infty$ and hence, also applying Lemma 1 to the equivalent term in $\Omega_{2}$, we can write

$$
\begin{gathered}
\delta H=\int_{\mathbb{R}} \int_{-h_{1}}^{\eta} \rho_{1}\left(\nabla \varphi_{1}\right) \cdot \nabla \delta \varphi_{1} d y d x+\int_{\mathbb{R}} \int_{\eta}^{h_{2}} \rho_{2}\left(\nabla \varphi_{2}\right) \cdot \nabla \delta \varphi_{2} d y d x \\
+\frac{1}{2} \int_{\mathbb{R}} \rho_{1}\left(\nabla \varphi_{1}\right)_{c}^{2} \delta \eta d x-\frac{1}{2} \int_{\mathbb{R}} \rho_{2}\left(\nabla \varphi_{2}\right)_{c}^{2} \delta \eta d x+\int_{\mathbb{R}}\left(\rho_{1}-\rho_{2}\right) g \eta \delta \eta d x \\
+\int_{\mathbb{R}} \rho_{1} \omega_{1} \eta \partial_{x} \eta\left(\delta \varphi_{1}\right)_{c} d x-\int_{\mathbb{R}} \rho_{2} \omega_{2} \eta \partial_{x} \eta\left(\delta \varphi_{2}\right)_{c} d x \\
-\int_{\mathbb{R}}\left(\rho_{1} \omega_{1} \eta\left(\partial_{x} \varphi_{1}\right)_{c}-\rho_{2} \omega_{2} \eta\left(\partial_{x} \varphi_{2}\right)_{c}\right) \delta \eta d x+\frac{1}{2} \int_{\mathbb{R}}\left(\rho_{1} \omega_{1}^{2} \eta^{2}-\rho_{2} \omega_{2}^{2} \eta^{2}\right) \delta \eta d x .
\end{gathered}
$$

Gauss' theorem is used to expand the following $\left(\Omega_{1}\right)$ term in terms of the interface and the upper and lower boundary normals, noting that the variation in the velocity potentials at the boundaries, $\left(\partial \varphi_{1}\right)_{b}$ and $\left(\partial \varphi_{2}\right)_{l}$, are zero:

$$
\int_{\mathbb{R}} \int_{-h_{1}}^{\eta}\left(\nabla \varphi_{1}\right) \cdot \nabla \delta \varphi_{1} d y d x=\int_{\mathbb{R}}\left(\left(\partial_{y} \varphi_{1}\right)_{c}-\left(\partial_{x} \varphi_{1}\right)_{c} \partial_{x} \eta\right)\left(\delta \varphi_{1}\right)_{c} d x
$$


Also expanding the equivalent term in $\Omega_{2}$ gives

$$
\begin{aligned}
\delta H= & \int_{\mathbb{R}} \rho_{1}\left(\left(\partial_{y} \varphi_{1}\right)_{c}-\left(\partial_{x} \varphi_{1}\right)_{c} \partial_{x} \eta\right)\left(\delta \varphi_{1}\right)_{c} d x \\
& +\int_{\mathbb{R}} \rho_{2}\left(\left(\partial_{y} \varphi_{2}\right)_{c}-\left(\partial_{x} \varphi_{2}\right)_{c} \partial_{x} \eta\right)\left(\delta \varphi_{2}\right)_{c} d x+\frac{1}{2} \int_{\mathbb{R}} \rho_{1}\left(\nabla \varphi_{1}\right)_{c}^{2} \delta \eta d x \\
- & \frac{1}{2} \int_{\mathbb{R}} \rho_{2}\left(\nabla \varphi_{2}\right)_{c}^{2} \delta \eta d x+\int_{\mathbb{R}}\left(\rho_{1}-\rho_{2}\right) g \eta \delta \eta d x+\int_{\mathbb{R}} \rho_{1} \omega_{1} \eta \partial_{x} \eta\left(\delta \varphi_{1}\right)_{c} d x \\
- & \int_{\mathbb{R}} \rho_{2} \omega_{2} \eta \partial_{x} \eta\left(\delta \varphi_{2}\right)_{c} d x-\int_{\mathbb{R}}\left(\rho_{1} \omega_{1} \eta\left(\partial_{x} \varphi_{1}\right)_{c}-\rho_{2} \omega_{2} \eta\left(\partial_{x} \varphi_{2}\right)_{c}\right) \delta \eta d x \\
& +\frac{1}{2} \int_{\mathbb{R}}\left(\rho_{1} \omega_{1}^{2} \eta^{2}-\rho_{2} \omega_{2}^{2} \eta^{2}\right) \delta \eta d x .
\end{aligned}
$$

Next, we express the variation in the interface velocity potential as

$$
\begin{aligned}
\delta\left(\left(\varphi_{i}\right)_{c}\right) & =\left(\partial_{y} \varphi_{i}\right)_{c} \delta \eta+\left(\delta \varphi_{i}\right)_{c} \\
\Rightarrow \quad\left(\delta \varphi_{i}\right)_{c} & =\delta \xi_{i}-\left(\partial_{y} \varphi_{i}\right)_{c} \delta \eta,
\end{aligned}
$$

and hence the varied Hamiltonian can therefore be written as

$$
\begin{gathered}
\delta H=\int_{\mathbb{R}}\left[-\rho_{1}\left(\partial_{y} \varphi_{1}\right)_{c}\left(\left(\partial_{y} \varphi_{1}\right)_{c}-\left(\partial_{x} \varphi_{1}\right)_{c} \partial_{x} \eta\right)+\rho_{2}\left(\partial_{y} \varphi_{2}\right)_{c}\left(\left(\partial_{y} \varphi_{2}\right)_{c}-\left(\partial_{x} \varphi_{2}\right)_{c} \partial_{x} \eta\right)\right. \\
+\frac{1}{2} \rho_{1}\left(\nabla \varphi_{1}\right)_{c}^{2}-\frac{1}{2} \rho_{2}\left(\nabla \varphi_{2}\right)_{c}^{2}+\left(\rho_{1}-\rho_{2}\right) g \eta-\rho_{1} \omega_{1} \eta\left(\partial_{y} \varphi_{1}\right)_{c} \partial_{x} \eta+\rho_{2} \omega_{2} \eta\left(\partial_{y} \varphi_{2}\right)_{c} \partial_{x} \eta \\
\left.-\rho_{1} \omega_{1} \eta\left(\partial_{x} \varphi_{1}\right)_{c}+\rho_{2} \omega_{2} \eta\left(\partial_{x} \varphi_{2}\right)_{c}+\frac{1}{2} \rho_{1} \omega_{1}^{2} \eta^{2}-\frac{1}{2} \rho_{2} \omega_{2}^{2} \eta^{2}\right] \delta \eta d x \\
+\int_{\mathbb{R}} \rho_{1}\left[\omega_{1} \eta \partial_{x} \eta+\left(\partial_{y} \varphi_{1}\right)_{c}-\left(\partial_{x} \varphi_{1}\right)_{c} \partial_{x} \eta\right] \delta \xi_{1} d x \\
-\int_{\mathbb{R}} \rho_{2}\left[\omega_{2} \eta \partial_{x} \eta+\left(\partial_{y} \varphi_{2}\right)_{c}-\left(\partial_{x} \varphi_{2}\right)_{c} \partial_{x} \eta\right] \delta \xi_{2} d x
\end{gathered}
$$


Fixing $\xi_{1}$ and $\xi_{2}$ we can see that the functional derivative of the Hamiltonian with respect to the interface function, $\eta$, is

$$
\begin{array}{r}
\delta_{\eta} H=-\rho_{1}\left(\partial_{y} \varphi_{1}\right)_{c}\left(\left(\partial_{y} \varphi_{1}\right)_{c}-\left(\partial_{x} \varphi_{1}\right)_{c} \partial_{x} \eta\right)+\rho_{2}\left(\partial_{y} \varphi_{2}\right)_{c}\left(\left(\partial_{y} \varphi_{2}\right)_{c}-\left(\partial_{x} \varphi_{2}\right)_{c} \partial_{x} \eta\right) \\
+\frac{1}{2} \rho_{1}\left(\nabla \varphi_{1}\right)_{c}^{2}-\frac{1}{2} \rho_{2}\left(\nabla \varphi_{2}\right)_{c}^{2}+\left(\rho_{1}-\rho_{2}\right) g \eta-\rho_{1} \omega_{1} \eta\left(\partial_{y} \varphi_{1}\right)_{c} \partial_{x} \eta+\rho_{2} \omega_{2} \eta\left(\partial_{y} \varphi_{2}\right)_{c} \partial_{x} \eta \\
-\rho_{1} \omega_{1} \eta\left(\partial_{x} \varphi_{1}\right)_{c}+\rho_{2} \omega_{2} \eta\left(\partial_{x} \varphi_{2}\right)_{c}+\frac{1}{2} \rho_{1} \omega_{1}^{2} \eta^{2}-\frac{1}{2} \rho_{2} \omega_{2}^{2} \eta^{2}
\end{array}
$$

Next, using the replacement

$$
\frac{1}{2} \rho_{i}\left(\nabla \psi_{i}\right)_{c}^{2}=\frac{1}{2} \rho_{i}\left(\nabla \varphi_{i}\right)_{c}^{2}-\rho_{i} \omega_{i} \eta\left(\partial_{x} \varphi_{i}\right)_{c}+\frac{1}{2} \rho_{i} \omega_{i}^{2} \eta^{2}
$$

we rewrite (40) as

$$
\begin{gathered}
\delta_{\eta} H=-\rho_{1}\left(\partial_{y} \varphi_{1}\right)_{c}\left(\left(\partial_{y} \varphi_{1}\right)_{c}-\left(\partial_{x} \varphi_{1}\right)_{c} \partial_{x} \eta\right)+\rho_{2}\left(\partial_{y} \varphi_{2}\right)_{c}\left(\left(\partial_{y} \varphi_{2}\right)_{c}-\left(\partial_{x} \varphi_{2}\right)_{c} \partial_{x} \eta\right) \\
+\frac{1}{2} \rho_{1}\left(\nabla \psi_{1}\right)_{c}^{2}-\frac{1}{2} \rho_{2}\left(\nabla \psi_{2}\right)_{c}^{2}+\left(\rho_{1}-\rho_{2}\right) g \eta-\rho_{1} \omega_{1} \eta\left(\partial_{y} \varphi_{1}\right)_{c} \partial_{x} \eta+\rho_{2} \omega_{2} \eta\left(\partial_{y} \varphi_{2}\right)_{c} \partial_{x} \eta .
\end{gathered}
$$

Using the Bernoulli condition (9) we make the replacement

$$
\begin{aligned}
\frac{1}{2} \rho_{1}\left(\nabla \psi_{1}\right)_{c}^{2}-\frac{1}{2} \rho_{2}\left(\nabla \psi_{2}\right)_{c}^{2} & +\left(\rho_{1}-\rho_{2}\right) g \eta \\
& =-\rho_{1}\left(\partial_{t} \varphi_{1}\right)_{c}+\rho_{2}\left(\partial_{t} \varphi_{2}\right)_{c}+\rho_{1} \omega_{1} \chi_{1}-\rho_{2} \omega_{2} \chi_{2}
\end{aligned}
$$

which means we can rewrite (41) as

$$
\begin{array}{r}
\delta_{\eta} H=-\rho_{1}\left(\partial_{y} \varphi_{1}\right)_{c}\left(\left(\partial_{y} \varphi_{1}\right)_{c}-\left(\partial_{x} \varphi_{1}\right)_{c} \partial_{x} \eta\right)+\rho_{2}\left(\partial_{y} \varphi_{2}\right)_{c}\left(\left(\partial_{y} \varphi_{2}\right)_{c}-\left(\partial_{x} \varphi_{2}\right)_{c} \partial_{x} \eta\right) \\
-\rho_{1}\left(\partial_{t} \varphi_{1}\right)_{c}+\rho_{2}\left(\partial_{t} \varphi_{2}\right)_{c}-\rho_{1} \omega_{1} \eta\left(\partial_{y} \varphi_{1}\right)_{c} \partial_{x} \eta+\rho_{2} \omega_{2} \eta\left(\partial_{y} \varphi_{2}\right)_{c} \partial_{x} \eta+\rho_{1} \omega_{1} \chi_{1}-\rho_{2} \omega_{2} \chi_{2} .
\end{array}
$$

Recalling the kinematic boundary condition in (10) and multiplying across by $\left(\partial_{y} \varphi_{i}\right)_{c}$ gives

$$
\partial_{t} \eta\left(\partial_{y} \varphi_{i}\right)_{c}=\left(\partial_{y} \varphi_{i}\right)_{c}^{2}-\left(\partial_{x} \varphi_{i}\right)_{c}\left(\partial_{y} \varphi_{i}\right)_{c} \partial_{x} \eta+\omega_{i} \eta\left(\partial_{y} \varphi_{i}\right)_{c} \partial_{x} \eta,
$$

which means we can rewrite (43) as

$$
\begin{aligned}
\delta_{\eta} H=-\rho_{1} \partial_{t} \eta\left(\partial_{y} \varphi_{1}\right)_{c}+\rho_{2} \partial_{t} \eta\left(\partial_{y} \varphi_{2}\right)_{c}-\rho_{1}\left(\partial_{t} \varphi_{1}\right)_{c} & +\rho_{2}\left(\partial_{t} \varphi_{2}\right)_{c} \\
& +\rho_{1} \omega_{1} \chi_{1}-\rho_{2} \omega_{2} \chi_{2} .
\end{aligned}
$$


From (38) we use $\partial_{t} \xi_{i}=\left(\partial_{t} \varphi_{i}\right)_{c}+\left(\partial_{y} \varphi_{i}\right)_{c} \partial_{t} \eta$, therefore

$$
\delta_{\eta} H=-\rho_{1} \partial_{t} \xi_{1}+\rho_{2} \partial_{t} \xi_{2}+\rho_{1} \omega_{1} \chi_{1}-\rho_{2} \omega_{2} \chi_{2} .
$$

Noting that $\xi:=\rho_{1} \xi_{1}-\rho_{2} \xi_{2}$ this gives

$$
\delta_{\eta} H=-\partial_{t} \xi+\rho_{1} \omega_{1} \chi_{1}-\rho_{2} \omega_{2} \chi_{2} .
$$

At the interface, using (2), we can define the velocity components in terms of the stream function for $\Omega_{1}$ as

$$
\left\{\begin{array}{l}
\left(u_{1}\right)_{c}=-\left(\partial_{y} \psi_{1}\right)_{c} \\
\left(v_{1}\right)_{c}=\left(\partial_{x} \psi_{1}\right)_{c}
\end{array}\right.
$$

and for $\Omega_{2}$ as

$$
\left\{\begin{array}{l}
\left(u_{2}\right)_{c}=-\left(\partial_{y} \psi_{2}\right)_{c} \\
\left(v_{2}\right)_{c}=\left(\partial_{x} \psi_{2}\right)_{c} .
\end{array}\right.
$$

However, at any moment in time any arbitrary point $(x, y)$ at the interface will be moving at a distinct velocity which can be measured independent of knowing the vorticities or velocity potentials, i.e. $\left(u_{1}\right)_{c}=\left(u_{2}\right)_{c}$ and $\left(v_{1}\right)_{c}=$ $\left(v_{2}\right)_{c}$, therefore

$$
\left\{\begin{array}{l}
\left(\partial_{y} \psi_{1}\right)_{c}=\left(\partial_{y} \psi_{2}\right)_{c} \\
\left(\partial_{x} \psi_{1}\right)_{c}=\left(\partial_{x} \psi_{2}\right)_{c}
\end{array}\right.
$$

i.e.

$$
\left(\nabla \psi_{1}\right)_{c}=\left(\nabla \psi_{2}\right)_{c}
$$

which means that $\left(\psi_{1}\right)_{c}$ and $\left(\psi_{2}\right)_{c}$ differ only by a constant. As potentials are modulo an additive constant, again using assumption (3), as $|x| \rightarrow \infty$ then $\chi_{i}$ goes to zero as $\chi_{i}=\chi_{i}(\eta)$ and hence $\left(\psi_{1}\right)_{c}$ and $\left(\psi_{2}\right)_{c}$ are equal, i.e.

$$
\chi_{1}=\chi_{2},
$$

i.e. it is a natural physical fact that there is no flow through the common interface. We define $\chi:=\chi_{1}=\chi_{2}$. Using this result, and introducing $\omega:=\rho_{1} \omega_{1}-\rho_{2} \omega_{2},(47)$ can be written as

$$
\partial_{t} \xi=-\delta_{\eta} H+\omega \chi
$$


Recalling the varied Hamiltonian (39) and fixing $\eta$ and using, from the kinematic boundary conditions (10),

$$
\partial_{t} \eta=\partial_{x} \eta \omega_{i} \eta-\partial_{x} \eta\left(\partial_{x} \varphi_{i}\right)_{c}+\left(\partial_{y} \varphi_{i}\right)_{c}
$$

we get

$$
\left.\delta H\right|_{\delta \eta=0}=\int_{\mathbb{R}} \rho_{1} \partial_{t} \eta \delta \xi_{1} d x-\int_{\mathbb{R}} \rho_{2} \partial_{t} \eta \delta \xi_{2} d x=\int_{\mathbb{R}} \partial_{t} \eta\left(\rho_{1} \delta \xi_{1}-\rho_{2} \delta \xi_{2}\right) d x .
$$

Therefore, recalling that $\xi=\rho_{1} \xi_{1}-\rho_{2} \xi_{2}$ and as $\delta$ is additive $\delta \xi=\rho_{1} \delta \xi_{1}-\rho_{2} \delta \xi_{2}$ this means

$$
\delta H=\int_{\mathbb{R}}\left(-\partial_{t} \xi+\omega \chi\right) \delta \eta d x+\int_{\mathbb{R}} \partial_{t} \eta \delta \xi d x
$$

which gives the non-canonical system

$$
\left\{\begin{array}{l}
\partial_{t} \xi=-\delta_{\eta} H+\omega \chi \\
\partial_{t} \eta=\delta_{\xi} H
\end{array}\right.
$$

In order to prove that this system has Hamiltonian form we first derive the following lemma.

\section{Lemma 2.}

$$
\chi(x, t)=\int_{-\infty}^{x} \frac{\delta H}{\delta \xi\left(x^{\prime}\right)} d x^{\prime}
$$

Proof. From (10)

$$
\partial_{t} \eta=\partial_{x} \eta\left(\partial_{y} \psi_{i}\right)_{c}+\left(\partial_{x} \psi_{i}\right)_{c}
$$

but, on the other hand (considering $t$ as a parameter)

$$
\frac{d}{d x} \chi(x, t)=\frac{d}{d x} \psi_{i}(x, \eta(x, t), t)=\left(\partial_{x} \psi_{i}\right)_{c}+\partial_{\eta} \psi_{i} \partial_{x} \eta=\left(\partial_{x} \psi_{i}\right)_{c}+\left(\partial_{y} \psi_{i}\right)_{c} \partial_{x} \eta
$$


Therefore, by comparing (58) and (59) (noting the following remark)

$$
\chi(x, t)=\int_{-\infty}^{x} \partial_{t} \eta\left(x^{\prime}, t\right) d x^{\prime}
$$

But, from (56)

$$
\partial_{t} \eta\left(x^{\prime}, t\right)=\frac{\delta H}{\delta \xi\left(x^{\prime}\right)}
$$

thus proving the lemma.

Remark. From the assumptions given by (3) and (4)

$$
\lim _{|x| \rightarrow \infty}\left(\psi_{i}(x, y, t)\right)_{c}=0 \Rightarrow \lim _{|x| \rightarrow \infty} \chi(x, t)=0 .
$$

Corollary 1. From Lemma 2

$$
\int_{\mathbb{R}} \partial_{t} \eta(x, t) d x=0
$$

and therefore

$$
\int_{\mathbb{R}} \eta(x, t) d x=\text { constant. }
$$

Remark. To calculate $\bar{\eta}$, the average of $\eta$, the above constant will be divided by an infinite length therefore $\bar{\eta}=0$.

Therefore we can write (56) as:

$$
\left\{\begin{array}{l}
\partial_{t} \xi=-\delta_{\eta} H+\omega \int_{-\infty}^{x} \frac{\delta H}{\delta \xi\left(x^{\prime}\right)} d x^{\prime} \\
\partial_{t} \eta=\delta_{\xi} H
\end{array}\right.
$$

From Wahlén [17] we know that this system of equations has a Hamiltonian form

$$
\left\{\begin{array}{l}
\partial_{t} \xi=\{\xi, H\} \\
\partial_{t} \eta=\{\eta, H\}
\end{array}\right.
$$

where the Poisson bracket in [19] is defined as

$$
\{A, B\}=\int_{\mathbb{R}}\left(\frac{\delta A}{\delta \eta(x)} \frac{\delta B}{\delta \xi(x)}-\frac{\delta A}{\delta \xi(x)} \frac{\delta B}{\delta \eta(x)}\right) d x+\omega \int_{\mathbb{R}}\left(\frac{\delta A}{\delta \xi(x)} \int_{0}^{x} \frac{\delta B}{\delta \xi\left(x^{\prime}\right)} d x^{\prime}\right) d x .
$$

One can verify that (67) satisfies all properties of a Poisson bracket. However, this fact will be established by other means in the next section. 


\section{Canonical Hamiltonian Equations of Motion}

The velocity potential at the interface, $\xi$, is defined modulo an additive constant. We chose to transform it, as per the single media case [19], to a new variable $\zeta$ as follows

$$
\xi \rightarrow \zeta=\xi-\frac{\omega}{2} \int_{-\infty}^{x} \eta\left(x^{\prime}, t\right) d x^{\prime}
$$

We show the system under study has canonical Hamiltonian structure by the following theorem:

Theorem 1. The system under study is a canonical Hamiltonian system described by the phase space variables $\eta$ and $\zeta$.

Proof. From (55):

$$
\delta H=\int_{\mathbb{R}}\left(-\partial_{t} \xi+\omega \chi\right) \delta \eta d x+\int_{\mathbb{R}} \partial_{t} \eta \delta \xi d x
$$

and applying the variable transformation given by (68) gives

$$
\begin{aligned}
& \delta H= \\
& \int_{\mathbb{R}}\left(-\partial_{t} \zeta-\frac{\omega}{2} \int_{-\infty}^{x} \partial_{t} \eta\left(x^{\prime}, t\right) d x^{\prime}+\omega \chi\right) \delta \eta(x) d x+\int_{\mathbb{R}} \partial_{t} \eta\left(\delta \zeta+\frac{\omega}{2} \int_{-\infty}^{x} \delta \eta\left(x^{\prime}\right) d x^{\prime}\right) d x .
\end{aligned}
$$

Using Lemma 2 gives

$$
\delta H=\int_{\mathbb{R}}\left(-\partial_{t} \zeta+\frac{\omega}{2} \chi\right) \delta \eta(x) d x+\int_{\mathbb{R}} \partial_{t} \eta\left(\delta \zeta+\frac{\omega}{2} \int_{-\infty}^{x} \delta \eta\left(x^{\prime}\right) d x^{\prime}\right) d x .
$$

Next, using integration by parts we can write

$$
\begin{aligned}
\int_{\mathbb{R}} \partial_{t} \eta\left(\int_{-\infty}^{x} \delta \eta\left(x^{\prime}\right) d x^{\prime}\right) d x= & {\left[\int_{-\infty}^{x} \delta \eta\left(x^{\prime}\right) d x^{\prime} \int_{-\infty}^{x} \partial_{t} \eta\left(x^{\prime \prime}, t\right) d x^{\prime \prime}\right]_{-\infty}^{+\infty} } \\
& -\int_{\mathbb{R}}\left(\int_{-\infty}^{x} \partial_{t} \eta\left(x^{\prime \prime}, t\right) d x^{\prime \prime}\right) \delta \eta(x) d x
\end{aligned}
$$


and, using Corollary 1 means the first term on the right-hand side is zero and applying Lemma 2 to the second term means we rewrite (71) as

$$
\delta H=\int_{\mathbb{R}}\left(-\partial_{t} \zeta+\frac{\omega}{2} \chi\right) \delta \eta d x+\int_{\mathbb{R}} \partial_{t} \eta \delta \zeta-\frac{\omega}{2} \int_{\mathbb{R}} \chi \delta \eta d x .
$$

Noting the cancellation of the $\omega \chi$ terms this gives the canonical Hamiltonian system

$$
\left\{\begin{array}{l}
\partial_{t} \zeta=-\delta_{\eta} H \\
\partial_{t} \eta=\delta_{\zeta} H
\end{array}\right.
$$

Thus, in general,

$$
\{A, B\}=\int_{\mathbb{R}}\left(\frac{\delta A}{\delta \eta(x)} \frac{\delta B}{\delta \zeta(x)}-\frac{\delta A}{\delta \zeta(x)} \frac{\delta B}{\delta \eta(x)}\right) d x .
$$

This indeed shows that (67) defines a (non-canonical) Poisson bracket in terms of the variables $(\eta, \xi)$.

Finally, using the transformation (68) we can write the Hamiltonian in terms of the conjugate variables $(\eta, \zeta)$ as

$$
\begin{aligned}
& H(\eta, \zeta)= \\
& \frac{1}{2} \int_{\mathbb{R}}\left[\zeta+\frac{\omega}{2} \int_{-\infty}^{x} \eta\left(x^{\prime}, t\right) d x^{\prime}\right]\left(G_{1}(\eta) B^{-1} G_{2}(\eta)\right)\left[\zeta+\frac{\omega}{2} \int_{-\infty}^{x} \eta\left(x^{\prime}, t\right) d x^{\prime}\right] d x \\
& +\frac{1}{2} \int_{\mathbb{R}}\left(\rho_{1}-\rho_{2}\right) g \eta^{2} d x-\frac{1}{2} \int_{\mathbb{R}} \rho_{1} \rho_{2}\left(\omega_{2}-\omega_{1}\right)^{2} \eta \partial_{x} \eta B^{-1} \eta \partial_{x} \eta d x \\
& +\int_{\mathbb{R}} \eta \partial_{x} \eta B^{-1}\left(\rho_{1} \omega_{1} G_{2}(\eta)\left[\zeta+\frac{\omega}{2} \int_{-\infty}^{x} \eta\left(x^{\prime}, t\right) d x^{\prime}\right]\right. \\
& \left.+\rho_{2} \omega_{2} G_{1}(\eta)\left[\zeta+\frac{\omega}{2} \int_{-\infty}^{x} \eta\left(x^{\prime}, t\right) d x^{\prime}\right]\right) d x+\frac{1}{6} \int_{\mathbb{R}}\left(\rho_{1} \omega_{1}^{2}-\rho_{2} \omega_{2}^{2}\right) \eta^{3} d x .
\end{aligned}
$$




\section{$7 \quad$ Some Further Remarks}

The case with periodic boundary conditions can be treated similarly to the problem with decaying to zero conditions at $\pm \infty$. The same results remain valid when all quantities take values in the class of periodic functions of period $L$. Then one can consider only a domain with $0<x<L$ and replace $\int_{\mathbb{R}} d x$ with $\int_{0}^{L} d x$. (68) changes into $\zeta=\xi-\frac{\omega}{2} \int_{0}^{x}\left(\eta\left(x^{\prime}, t\right)-\bar{\eta}\right) d x^{\prime}$ where $\bar{\eta}=\frac{1}{L} \int_{0}^{L} \eta(x, t) d x$ is the average of $\eta$. This is in order to preserve the periodicity, since the potential $\xi$ is determined up to a constant. The details are given in [19].

\section{Conclusion}

A closed water-wave system consisting of 2 immiscible media with constant (non-zero) vorticities and constant densities separated by a common free interface was considered where the upper and lower media are bound above and below, respectively, by impermeable boundaries. By examining nonlateral 2-dimensional flow, with gravitationally induced waves, the system governing equations were used to derive the Hamiltonian form of the system and the equations of motion in terms of phase space variables $(\eta, \xi)$ with noncanonical Hamiltonian structure. This was achieved by defining an interface velocity potential, $\xi$, in terms of the respective interface velocity potentials and constant densities of the 2 domains.

Moreover, by performing a variable transformation it was then shown that the system actually has canonical Hamiltonian structure with canonical phase space variables $(\eta, \zeta)$. Finally, the Hamiltonian of the system in terms of these phase space variables was given.

\section{Acknowledgements}

The author would like to thank R. Ivanov and E. Prodanov at the Dublin Institute of Technology for their invaluable discussions on matters relating to this article and also thank an anonymous referee for important suggestions which have contributed to improvements in the overall quality of the article. 


\section{References}

[1] V. Zakharov, Stability of periodic waves of finite amplitude on the surface of a deep fluid, Zh. Prikl. Mekh. Tekh. Fiz. 9 (1968) 86-94.

[2] T. Benjamin, P. Olver, Hamiltonian structure, symmetries and conservation laws for water waves, J. Fluid Mech. 125 (1982) 137-185.

[3] W. Craig, Water waves, Hamiltonian systems and Cauchy integrals, IMA Vol. Math. Appl. 30 (1991) 37-45.

[4] D. Milder, A note regarding 'On Hamilton's principle for water waves', J. Fluid Mech. 83 (1977) 159-161.

[5] J. Miles, Hamiltonian formulations for surface waves, Appl. Sci. Res. 37 (1981) 103-110.

[6] J. Miles, On Hamilton's principle for water waves, J. Fluid Mech. 83 (1977) 153-158.

[7] F. Gerstner, Theorie der Wellen samt einer daraus abgeleiteten Theorie der Deichprofile, Ann. Phys. 2 (1809) 412-445.

[8] A. Constantin, On the deep water wave motion, J. Phys. A 34 (2001) $1405-1417$.

[9] A. Constantin, J. Escher, Symmetry of steady periodic surface water waves with vorticity, J. Fluid Mech. 498 (2004) 171-181.

[10] A. Constantin, J. Escher, Symmetry of steady deep-water waves with vorticity, Eur. J. Appl. Math. 15 (2004) 755-768.

[11] A. Constantin, D. Sattinger, W. Strauss, Variational formulations for steady water waves with vorticity, J. Fluid Mech. 548 (2006) 151-163.

[12] A. Constantin, W. Strauss, Exact steady periodic water waves with vorticity, Comm.Pure Appl. Math. 57 (2004) 481-527.

[13] W. Craig, C. Sulem, Numerical simulation of gravity waves, J. Comp. Phys. 108 (1993) 73-83. 
[14] M. Ehrnström, Uniqueness for steady water waves with vorticity, Int. Math. Res.Not. 2005 (2005) 3721-3726.

[15] B. Kolev, D. Sattinger, Variational principles for water waves, SIAM J. Math.Anal. 38 (2006) 906-920.

[16] A. Teles da Silva, D. Peregrine, Steep, steady surface waves on water of finite depth with constant vorticity, J. Fluid Mech. 195 (1988) 281-302.

[17] E. Wahlén, Steady periodic capillary-gravity waves with vorticity, SIAM J. Math. Anal. 38 (2006) 921-943. doi:10.1007/s1151200600247.

[18] A. Constantin, R. Ivanov, E. Prodanov, Nearly-Hamiltonian Structure for Water Waves with Constant Vorticity, J. Math. Fluid Mech. 9 (2007) $1-14$.

[19] E. Wahlén, A Hamiltonian Formulation of Water Waves with Constant Vorticity, Lett. Math. Phys. 79 (2007) 303-315.

[20] T. Benjamin, T. Bridges, Reappraisal of the Kelvin-Helmholtz problem. Part 1. Hamiltonian structure, J. Fluid Mech. 333 (1997) 301-325.

[21] T. Benjamin, T. Bridges, Reappraisal of the Kelvin-Helmholtz problem. Part 2. Interaction of the Kelvin-Helmholtz, superharmonic and Benjamin-Feir instabilities, J. Fluid Mech. 333 (1997) 327-373.

[22] W. Craig, P. Guyenne, H. Kalisch, Hamiltonian long wave expansions for free surfaces and interfaces, Comm. Pure Appl. Math. 24 (2005) $1587-1641$.

[23] W. Craig, P. Guyenne, C. Sulem, Coupling between internal and surface waves, Nat. Hazards 57 (2011) 617-642. 